\title{
Effect of Oxygen Pneumoperitoneum on Hypoxia Tolerance in Beagle Dogs: An Experimental Study
}

\section{Jiangbei CAO}

Chinese PLA General Hospital

\section{Yongyi ZHANG}

1.NO. 211 hospital of PLA; 2.Chinese PLA General Hospital

\section{Wei WANG}

The General Hospital of The PLA Rocket Force

\section{Hao LI}

Chinese PLA General Hospital

\section{Weidong MI ( $\nabla$ wwdd1962@aliyun.com )}

Chinese PLA General Hospital https://orcid.org/0000-0001-9814-2450

\section{Research article}

Keywords: Airway management, Pneumoperitoneum, Artificial, Asphyxia, Cardiopulmonary Resuscitation

Posted Date: September 8th, 2019

DOI: https://doi.org/10.21203/rs.2.14085/v1

License: @ (i) This work is licensed under a Creative Commons Attribution 4.0 International License. Read Full License 


\section{Abstract}

Background: When an airway is difficult to manage during surgery, it can be fatal. Thus, peritoneum oxygenation may be theoretically feasible as an alternative route to supply oxygen. The objective of this study is to investigate whether artificial oxygen pneumoperitoneum combined with abdominal lifting and compression is effective in systemic oxygenation and hypoxia tolerance in a beagle dog model of asphyxia.

Methods: Latin Square design was adopted. Eight adult female beagle dogs were divided into four subgroups; they received four treatments in sequence in four sessions at 1-week intervals. The pre-treatment procedures included general anesthesia and a pause in ventilation to establish an asphyxia model. Each subgroup was subjected to the treatments as follows: negative control(A), artificial oxygen pneumoperitoneum(B), artificial oxygen pneumoperitoneum combined with abdominal lifting and compression from the 1st minute after asphyxia(C), or the same as $C$ but starting the method from the 5th minute(D). Vital signs, important organ functions, and arterial blood gas were monitored. Hypoxia tolerance was assessed by the asphyxia time (from pause to restoration of ventilation) and $\mathrm{PaO} 2$ levels at $0,6,7,8,9$, and 10 minutes after asphyxia onset.

Results: The physiological status of the beagles was approximately identical before each experiment and the treatments did not aggravate the impact of hypoxia. The asphyxia time for treatments A, B, C, and D were 9.65 $\pm 1.10 \mathrm{~min}, 11.18 \pm 0.50 \mathrm{~min}, 11.45 \pm 0.79 \mathrm{~min}$, and $11.70 \pm 0.99 \mathrm{~min}$, respectively; the time was lengthened in treatments $B, C$, and $D$, compared with that in treatment $A(P<0.01)$. Moreover, $P a 02$ levels at 9 and 10 min were higher in treatments $B, C$, and $D$ than in treatment $A(P<0.01)$.

Conclusions: Artificial oxygen pneumoperitoneum combined with abdominal lifting and compression appears safe and effective and may improve systemic oxygenation and enhance hypoxia tolerance.

\section{Background}

During emergency rescue and anesthesia procedures, maintaining the air way is a top priority, as prompt and efficient oxygen supply is required to sustain the patient's life. ${ }^{[1,2]}$ However, it can be challenging to administer oxygen when managing a difficult airway caused by morbid obesity, maxillofacial trauma, or tracheal tumors; life-threatening difficulties in oxygen administration can occur, especially when encountering an emergency airway-unanticipated difficulties in either intubation or ventilation ${ }^{[3,4]}$.Thus, extra-pulmonary oxygenation represents an important alternative route to supply oxygen ${ }^{[5-7]}$. Thus by far, extracorporeal membrane oxygenation, intravascular oxygenator, and percutaneous cardio-pulmonary support have been applied for extra-pulmonary oxygenation; however, these procedures are complex, time-consuming, laborintensive, and associated with many complications, due to multiple transfusions and systemic heparin administration ${ }^{[6-10]}$. Hence, researchers have attempted to find an easy, safe, and effective method by which clinicians can improve oxygen supply. 
The peritoneum is a biological semi-permeable membrane comprising a large, absorptive, highly permeable surface with rich vascular beds. Therefore, it may be used for extra-pulmonary oxygenation ${ }^{[11]}$. Previously, peritoneal oxygenation has been shown to moderately increase systemic arterial partial pressure of oxygen in rabbit, rat, and pig models ${ }^{[5,12-14]}$ via direct abdominal ventilation or intra-peritoneal infusion with oxygen carriers, such as perfluorocarbon, red blood cells, or oxygen micro-bubbles ${ }^{[5, ~ 8, ~ 12, ~ 13, ~ 15-19] . ~ H e n c e, ~ p e r i t o n e a l ~}$ oxygenation has long been desired for extra-pulmonary oxygenation, as it is easy to perform, compared to use of an extracorporeal circuit.

Based on the above findings, oxygenation status may be improved by artificial oxygen pneumoperitoneum. In cases of hypoxia, oxygen saturation in the splanchnic bed may be reduced considerably; hence, additional oxygen could be absorbed from the peritoneal cavity. Additionally, recent research has suggested that abdominal lifting and compression could promote blood circulation in abdominal viscera and facilitate breathing by enhancing diaphragm movement ${ }^{[20,21]}$. Thus, the objective of this study was to investigate the effect of artificial oxygen pneumoperitoneum combined with abdominal lifting and compression on systemic oxygen supply and hypoxia tolerance in a beagle dog model of asphyxia.

\section{Methods}

\subsection{Animals}

Healthy adult female beagle dogs $(n=8)$ weighing 8-10kg were purchased from the Academy of Military Medicine and Science of Chinese People's Liberation Army(PLA) (Beijing, China: Permit Number: SCXK(JUN) 2014-0001). All beagles were housed individually in accordance with guidelines for indoor pets at the Experimental Animal Unit of PLA General Hospital, and were given access to standard food and water at scheduled times in an air-conditioned room set at $24 \pm 1{ }^{\circ} \mathrm{C}$ with $50 \pm 10 \%$ humidity, under a standard 12-12 hour light-dark cycle(light on from 7 AM to 7 PM). Animals were housed in the same cage throughout the experimental period. Adequate measures were taken to minimize animal discomfort.

\subsection{Study Design}


We investigated four treatment modalities in this study:(A)control, in which dogs received abdominal puncture alone with a Veress needle, but no additional treatments after the asphyxia model;(B) pneumoperitoneum (Pneu), in which dogs received artificial oxygen pneumoperitoneum beginning at1 minute after the asphyxia model;(C) early pneumoperitoneum plus abdominal lifting and compression $(\mathrm{P}+1)$, in which dogs received artificial oxygen pneumoperitoneum combined with abdominal lifting and compression, which was started at 1 minute after asphyxia modeling began; (D) late pneumoperitoneum plus abdominal lifting and compression $(\mathrm{P}+5)$, in which dogs received artificial oxygen pneumoperitoneum combined with abdominal lifting and compression beginning at 5 minutes after asphyxia modeling was started. Beagle dogs were numbered by weight and then divided into four subgroups based on weightranging from light to heavy. During the 4-week experimental period, each dog within each subgroup received all four treatments in a different sequence from one another; between treatments, a 1-week washout period was used to allow physical recovery to baseline. To investigate the effects of experimental time, individual dog, and treatment modality, a $4 \times 4$ Latin Square design was utilized (Table 1).

\section{Table 1.Study Design Overview}

\begin{tabular}{|c|c|c|c|c|}
\hline & 1st week & 2nd week & 3rd week & 4th week \\
\hline Subgroup I & Treatment $\mathrm{A}$ & Treatment B & Treatment C & Treatment D \\
\hline (Dog1, Dog2) & (Con) & (Pneu) & $(P+1)$ & $(P+5)$ \\
\hline Subgroup II & Treatment $\mathrm{B}$ & Treatment $\mathrm{C}$ & Treatment D & Treatment A \\
\hline (Dog3, Dog4) & (Pneu) & $(P+1)$ & $(P+5)$ & \\
\hline Subgroup III & Treatment C & Treatment D & Treatment A & Treatment B \\
\hline (Dog5, Dog6) & $(P+1)$ & $(P+5)$ & (Con) & (Pneu) \\
\hline Subgroup IV & Treatment $\mathrm{D}$ & Treatment $\mathrm{A}$ & Treatment B & Treatment C \\
\hline (Dog7, Dog8) & $(P+5)$ & (Con) & (Pneu) & $(P+1)$ \\
\hline
\end{tabular}

Con: control group, Pneu: pneumoperitoneum group, $P+1$ :early pneumoperitoneum plus abdominal lifting and compression group, $P+5$ : late pneumoperitoneum plus abdominal lifting and compression group

\subsection{Experimental protocol}


All dogs were fasted for 8 hours and denied access to water for 2 hours before the experiment. After anesthetization with propofol $(6 \mathrm{mg} / \mathrm{kg})$, rocuronium $(1 \mathrm{mg} / \mathrm{kg})$, and sufentanil $(0.8 \mu \mathrm{g} / \mathrm{kg})$ via intravenous infusion, the dogs were placed on a surgical table in a supine position and then an endotracheal tube (ID7.5 mm) was inserted into the endotracheal. General anesthesia was maintained by continuous intravenous infusion of propofol $(12 \mathrm{mg} / \mathrm{kg} \cdot \mathrm{h})$ and rocuronium $(2 \mathrm{mg} / \mathrm{kg} \cdot \mathrm{h})$, and mechanical ventilation was maintained with $100 \%$ oxygen (tidal volume, 25ml/kg; respiratory rate, $16 \mathrm{bpm})$. The dogs' vital signs such as $\mathrm{SpO}_{2}$, heart rate(HR), invasive arterial blood pressure, and cardiac output(CO), were monitored continuously throughout the procedure(V24E, Philips Inc., Amsterdam, The Netherlands; Vigileo-FloTrac ${ }^{\mathrm{TM}}$ system, Edwards LifeSciences Inc., Irvine, CA, USA). After a baseline ventilation period of 5 minutes, the asphyxia model was established by clamping the endotracheal tube. The dogs were then subjected to treatments A, B, C, or D, as described above. The artificial oxygen pneumoperitoneum was established in accordance with the clinical practice used in laparoscopic surgery ${ }^{[22]}$, and the pressure was set at $12 \mathrm{cmH}_{2} \mathrm{O}$ (model: PG120, Aesculap Inc., Tuttlingen, Germany). Abdominal wall lifting and compression ${ }^{[23]}$ was performed at a frequency of 60bpm, a ratio of 1:1, and a strength of $50 \mathrm{~N}$. Arterial blood gas (ABG) analyses were performed at baseline and at $6,7,8,9$, and 10 minutes, respectively, after the endotracheal tube was clamped, as well as at the time of asphyxia termination(model: GEM Premier 3000, Instrumentation Laboratory, Lexington, MA, USA). Asphyxia was terminated by administering 100\% oxygen when one of the following criteria was met: 1) $\mathrm{SpO}_{2}$ was $<50 \%$, (2) mean arterial pressure(MAP) was twice the baseline value, or (3) malignant arrhythmia occurred ${ }^{[24]}$. Asphyxia time was recorded, as was recovery time-the time at which the vital signs were stabilized. Then, the pneumoperitoneum was deflated and anesthesia was discontinued. Dogs were returned to their cages upon extubation and awakening. Venous blood was collected before the experiment, as well as at 2 and 24 hours after the experiment, and was analyzed to assess hepatic and renal function, as well as oxidative stress-related enzymes. After a 1-week washout period, the dogs underwent the next round of the experiment, as shown in Table 1. After all rounds of the experiments, the dogs were euthanized. Placed in a quiet and comfortable position, the dogs were deeply anesthetized and monitored as above, and then euthanasia solution(Beuthanasia-D Special, $86 \mathrm{mg} / \mathrm{kg}$ ) was injected. Measures were taken to minimize the stress. 


\subsection{Statistical analysis}

All data were analyzed by an observer who was blinded to the study protocol. Statistical calculations were performed using SPSS 17.0 (SPSS, Inc., Chicago, IL, USA). Data were expressed as the mean \pm standard deviation. Intergroup comparisons were conducted using Latin Square ANOVA, followed by the least significant difference-t post hoc test to determine significant differences among treatments. Data regarding ABG analysis, HR, MAP, and other factors were analyzed using two-way ANOVA(treatment $\times$ trial time) with repeated measures followed by a Bonferroni post hoc test to analyze differences among treatment groups. P values $<0.05$ were considered statistically significant.

\section{Results}

\subsection{Physiological and biochemical statuses in each group were nearly identical before each round of the experiment}

As described in the experimental protocol, the dogs were subjected to a severe hypoxic attacknearly lethal-which resulted in damage to important organs, such as the heart, liver, and kidneys. Therefore, we included a 1-week interval between each round of the experiment to allow for physical recovery from the hypoxic attacks and to eliminate any lingering effects of the previous experiment. According to the Latin Square ANOVA results, all dogs were in a good state of health; no significant differences were observed among treatment groups with regard to $\mathrm{HR}$, MAP, $\mathrm{SpO}_{2}, \mathrm{CO}, \mathrm{PaO}_{2}, \mathrm{PaCO}_{2}, \mathrm{pH}$, other indexes of $\mathrm{ABG}$, or hepatic and renal functions before each round of experiments (Table 2). These findings suggested that the physiological and biochemical statuses of the dogs returned to baseline during the1-week intervals; additionally, there were no significant differences between experimental times or between individual dogs in terms of these indexes(Table 2). 


\begin{tabular}{|c|c|c|c|c|c|c|c|}
\hline Indexes & Con & Pneu & $\mathrm{P}+1$ & $\mathrm{P}+5$ & $P_{\text {treatment }}$ & $P_{\text {time }}$ & $P_{\text {dog }}$ \\
\hline HR(bpm) & $94 \pm 10.4$ & $96 \pm 16.4$ & $95 \pm 15.6$ & $94 \pm 14.2$ & 0.969 & 0.645 & 0.439 \\
\hline $\operatorname{MAP}(\mathrm{mmHg})$ & $86.6 \pm 5.24$ & $81.0 \pm 10.50$ & $83.0 \pm 10.42$ & $83.4 \pm 9.05$ & 0.225 & 0.228 & 0.062 \\
\hline $\mathrm{CO}(\mathrm{l} / \mathrm{min})$ & $8.10 \pm 0.76$ & $7.85 \pm 1.27$ & $8.55 \pm 1.11$ & $7.95 \pm 0.94$ & 0.509 & 0.164 & 0.383 \\
\hline $\mathrm{SpO}_{2}(\%)$ & $100 \pm 0$ & $100 \pm 0$ & $100 \pm 0$ & $100 \pm 0$ & - & - & - \\
\hline $\mathrm{PaO}_{2}(\mathrm{mmHg})$ & $587.00 \pm 43.55$ & $598.25 \pm 40.44$ & $578.38 \pm 48.77$ & $600.00 \pm 56.81$ & 0.780 & 0.379 & 0.463 \\
\hline $\mathrm{PaCO}_{2}(\mathrm{mmHg})$ & $33.13 \pm 2.850$ & $31.13 \pm 2.696$ & $32.00 \pm 2.390$ & $32.88 \pm 3.563$ & 0.439 & 0.075 & 0.405 \\
\hline $\mathrm{pH}$ & $7.411 \pm 0.038$ & $7.411 \pm 0.035$ & $7.410 \pm 0.030$ & $7.405 \pm 0.040$ & 0.964 & 0.660 & 0.114 \\
\hline $\mathrm{THbc}(\mathrm{g} / \mathrm{dl})$ & $11.06 \pm 0.64$ & $11.45 \pm 1.17$ & $11.49 \pm 0.68$ & $11.73 \pm 0.93$ & 0.487 & 0.118 & 0.571 \\
\hline $\mathrm{Na}^{+}(\mathrm{mmol} / \mathrm{l})$ & $145.75 \pm 1.39$ & $144.75 \pm 0.71$ & $145.38 \pm 1.60$ & $144.38 \pm 1.69$ & 0.209 & 0.101 & 0.697 \\
\hline $\mathrm{K}^{+}(\mathrm{mmol} / \mathrm{l})$ & $2.76 \pm 0.220$ & $2.71 \pm 0.327$ & $2.80 \pm 0.177$ & $2.75 \pm 0.239$ & 0.902 & 0.059 & 0.751 \\
\hline $\mathrm{Ca}^{++}(\mathrm{mmol} / \mathrm{l})$ & $1.18 \pm 0.023$ & $1.19 \pm 0.037$ & $1.22 \pm 0.034$ & $1.19 \pm 0.049$ & 0.251 & 0.219 & 0.699 \\
\hline $\operatorname{ALT}(I U / I)$ & $31.39 \pm 7.795$ & $28.84 \pm 11.839$ & $27.04 \pm 4.892$ & $29.60 \pm 7.419$ & 0.728 & 0.388 & 0.172 \\
\hline AST(IU/I) & $39.33 \pm 11.981$ & $34.35 \pm 7.197$ & $35.00 \pm 9.249$ & $40.10 \pm 6.983$ & 0.438 & 0.819 & 0.143 \\
\hline $\operatorname{Crea}(\mu \mathrm{mol} / \mathrm{l})$ & $49.29 \pm 8.115$ & $50.74 \pm 5.711$ & $52.11 \pm 5.499$ & $49.08 \pm 9.137$ & 0.736 & 0.098 & 0.103 \\
\hline $\operatorname{BUN}(\mathrm{mmol} / \mathrm{l})$ & $3.37 \pm 0.454$ & $3.44 \pm 0.535$ & $3.69 \pm 0.654$ & $3.36 \pm 0.465$ & 0.423 & 0.627 & 0.073 \\
\hline $\mathrm{Glu}(\mathrm{mmol} / \mathrm{l})$ & $4.54 \pm 0.555$ & $4.11 \pm 0.714$ & $4.60 \pm 0.576$ & $5.29 \pm 1.917$ & 0.305 & 0.427 & 0.932 \\
\hline $\mathrm{LDH}(\mathrm{IU} / \mathrm{I})$ & $141.4 \pm 47.08$ & $129.6 \pm 35.44$ & $145.8 \pm 55.13$ & $128.1 \pm 31.21$ & 0.225 & 0.591 & 0.063 \\
\hline GGT(IU/I) & $2.975 \pm 0.492$ & $2.862 \pm 0.447$ & $3.188 \pm 0.710$ & $3.350 \pm 0.678$ & 0.727 & 0.427 & 0.560 \\
\hline CK-MB(IU/I) & $322.33 \pm 38.32$ & $294.70 \pm 27.79$ & $308.93 \pm 26.48$ & $318.20 \pm 26.66$ & 0.317 & 0.612 & 0.460 \\
\hline $\mathrm{SOD}(\mathrm{IU} / \mathrm{ml})$ & $130.28 \pm 33.34$ & $145.91 \pm 25.61$ & $150.33 \pm 33.05$ & $138.65 \pm 38.51$ & 0.679 & 0.420 & 0.736 \\
\hline $\mathrm{MAO}(\mathrm{IU} / \mathrm{ml})$ & $3.925 \pm 1.025$ & $3.500 \pm 1.232$ & $3.688 \pm 0.958$ & $3.600 \pm 0.853$ & 0.833 & 0.676 & 0.144 \\
\hline Hct(\%) & $31.25 \pm 2.435$ & $32.50 \pm 6.164$ & $31.75 \pm 3.576$ & $33.86 \pm 4.190$ & 0.688 & 0.242 & 0.935 \\
\hline $\mathrm{HCO}_{3}{ }^{-} \mathrm{std}(\mathrm{mmol} / \mathrm{l})$ & $21.94 \pm 1.570$ & $22.09 \pm 1.229$ & $22.76 \pm 0.656$ & $22.18 \pm 1.091$ & 0.958 & 0.694 & 0.689 \\
\hline $\mathrm{Lac}(\mathrm{mmol} / \mathrm{l})$ & $0.313 \pm 0.083$ & $0.375 \pm 0.128$ & $0.413 \pm 0.146$ & $0.400 \pm 0.131$ & 0.325 & 0.631 & 0.110 \\
\hline $\mathrm{BE}(\mathrm{mmol} / \mathrm{l})$ & $-3.96 \pm 1.859$ & $-3.85 \pm 2.088$ & $-2.68 \pm 1.787$ & $-2.94 \pm 2.001$ & 0.416 & 0.677 & 0.170 \\
\hline
\end{tabular}

Data are presented as mean $\pm \mathrm{SD}$. $P_{\text {treatment }}$ indicates $P$ values of inter-groups comparison; $P_{\text {time }}$ indicates $P$ values of inter experimental time comparison; $P_{\text {dog }}$ indicates $P$ values of individuals of beagles comparison.

Con: control group, Pneu: pneumoperitoneum group, $P+1$ :early pneumoperitoneum plus abdominal lifting and compression group, $P+5$ : late pneumoperitoneum plus abdominal lifting and compression group

ALT, alanine aminotransferase; AST, aspartate aminotransferase; Crea, serum creatinine; BUN, blood urea nitrogen; Glu, blood glucose; LDH, lactate dehydrogenase; GGT, $\gamma$-glutamyl transpeptidase; CK-MB, creatine kinase isoenzymesB; SOD, superoxide dismutase; MAO, monoamine oxidase; Hct, red blood cell specific volume; Lac, lactate; BE, buffer exces 


\section{2. $\mathrm{PaO}_{2}$ in each group was approximately identical at the time of asphyxia termination}

It was critical to determine when to restore mechanical ventilation during the asphyxia model. We aimed to achieve the maximum possible extent at which the dogs exhibited the greatest tolerance to hypoxia. If restoration of mechanical ventilation was performed too early, the effect of artificial oxygen pneumoperitoneum on hypoxia tolerance could not be fully explored. If it was performed too late, hypoxia could cause irreversible damage or lead to failed resuscitation. $\mathrm{PaO}_{2}$ measurement is undoubtedly the gold standard for assessing internal oxygen content. However, ABG analysis could only be performed intermittently; thus, we used continuous indexes. Based on pre-experimental observations, we restored mechanical ventilation when one of the following conditions was met: 1$) \mathrm{SpO}_{2}$ was $<50 \%$, continually; 2) MAP was twice the baseline value; or 3) malignant arrhythmia occurred. We simultaneously performed ABG testing to measure $\mathrm{PaO}_{2}$ at the termination time. The above practices were implemented because: 1) occasionally, $\mathrm{SpO}_{2}$ could not be read due to poor peripheral circulation caused by severe hypoxia; 2) CO was reliable when hemodynamic status was stable, but could not be measured during severe fluctuations in hemodynamic status due to hypoxia; and 3) dogs exhibited different levels of intolerance to hypoxia in each experiment, indicating that HR or MAP alone could not accurately reflect pathological progress. Therefore, we combined these indexes to determine an accurate and reversible pathological point. Consistent with our pre-experiments, $\mathrm{PaO}_{2}$ measurements were not significantly different among groups when asphyxia was terminated $\left(P_{\text {treatment }}=0.762, P_{\text {time }}=0.700, P_{\text {dog }}=0.960\right.$, Fig. 1A), indicating that hypoxic states were similar among dogs at this time.

\subsection{Asphyxia time was lengthened by artificial oxygen pneumoperitoneum combined with abdominal lifting and compression}

As previously described, the dogs were subjected to multiple rounds of hypoxic states during the study period, which is harmful; however, this could be regarded as hypoxia tolerance training. Thus, we assessed whether the dogs showed enhanced tolerance to hypoxia in the later stages of the experiment, using Latin Square design. Notably, the effect of experimental time on 
hypoxia tolerance was not statistically significant $\left(\mathrm{P}_{\text {time }}=0.247\right)$;individual dogs also did not affect hypoxia tolerance $\left(\mathrm{P}_{\mathrm{dog}}=0.633\right)$. Asphyxia times of the Control, $\mathrm{Pneu}, \mathrm{P}+1$, and $\mathrm{P}+5$ treatment groups were $9.65 \pm 1.10 \mathrm{~min}, 11.18 \pm 0.50 \mathrm{~min}, 11.45 \pm 0.79 \mathrm{~min}$, and $11.70 \pm 0.99 \mathrm{~min}$, respectively (Fig. 1C). Artificial oxygen pneumoperitoneum combined with abdominal lifting and compression lengthened asphyxia time (Pneu vs Control, mean difference 1.52min [95\% confidence interval (95\%CI) 0.59, 2.45; $\mathrm{P}=0.003$ ]; $\mathrm{P}+1$ vs Control, mean difference 1.80min [95\%CI 0.87, 2.72; P=0.001]; P+5 vs Control, mean difference 2.01 min [95\%CI 1.12, 2.97; $\mathrm{P}<0.001$ ]; Fig. 1B and 1C). Finally, no significant differences were present in recovery time among groups(Fig. 1D).

\subsection{Artificial oxygen pneumoperitoneum combined with abdominal lifting and compression increased $\mathrm{PaO}_{2}$}

$\mathrm{PaO}_{2}$ is a reliable indicator of oxygen content. Based on our pre-experiments, $\mathrm{PaO}_{2}$ declined at a similar velocity among groups during the first 5 minutes of asphyxia, and the termination of asphyxia standard was met in some dogs after 10 minutes of asphyxia. Hence, $\mathrm{PaO}_{2}$ and $\mathrm{PaCO}_{2}$ were detected and compared at every minute beginning from the 6th to the 10th minute in this study (Fig. 2A and 2D). In Figure 2A and 2D, repeated measures two-way ANOVA revealed a difference in $\mathrm{PaO}_{2}$ levels among groups $(\mathrm{P}<0.05)$, and no difference in $\mathrm{PaCO}_{2}$ levels among groups $(\mathrm{P}>0.05) . \mathrm{PaO}_{2}$ reduction in the Control group was greater than that in other groups beginning from8 minutes after asphyxia; $\mathrm{PaCO}_{2}$ increased in a nearly linear fashion in all groups(Fig. 2A and 2D). Accordingly, we compared $\mathrm{PaO}_{2}$ values at the 9th and 10th minutes (Fig. 2B and 2C) and found that $\mathrm{PaO}_{2}$ levels in the Pneu, $\mathrm{P}+1$, and $\mathrm{P}+5$ groups were higher than that of the Control group $\left(\mathrm{PaO}_{2}\right.$, 9th min: Pneu vs Control, 8.50mmHg [95\%CI 3.50, 13.50]; P+1 vs Control, 11.5mmHg [95\%CI 6.50, 16.50]; P+5 vs Control, 15.00mmHg [95\% CI 10.00, 20.00]; 10th min: Pneu vs Control, 9.75mmHg [95\%CI 6.34, 13.16]; P+1 vs Control, 10.50mmHg [95\%CI 7.09, 13.91]; $\mathrm{P}+5$ vs Control, $13.25 \mathrm{mmHg}[95 \% \mathrm{CI} 9.84,16.67])$; additionally, $\mathrm{PaO}_{2}$ levels in the $\mathrm{P}+5$ group were higher than that in the Pneu group $\left(\mathrm{PaO}_{2}, 9\right.$ th min: $\mathrm{P}+5$ vs Pneu, $6.50 \mathrm{mmHg}$ [95\%CI 1.50, 11.50]; 10th min: P+5 vs Pneu, 3.50mmHg [95\%CI 0.09, 6.91];). These findings 
suggested that artificial oxygen pneumoperitoneum could increase $\mathrm{PaO}_{2}$ levels and that abdominal lifting and compression could further increase $\mathrm{PaO}_{2}$ levels during asphyxia.

\subsection{Changes in HR and MAP during asphyxia}

To investigate the safety and effect of this method on hemodynamics, we compared changes in HR and MAP in each group every 2 minutes, from baseline to the 10th min after asphyxia(Fig. 3). HR appeared to initially accelerate, then slowed(Fig.3A). No differences were observed among the groups (Fig. 3A and 3C), although HR in the Control group tended to be lower than that in other groups at $10 \mathrm{~min}$ (Fig. $3 \mathrm{~A}$ and $3 \mathrm{C}$ ). MAP appeared to increase in the Pneu, $\mathrm{P}+1$, and P+5 groups; in the Control group, it tended to initially increase, then decrease. Although no differences were observed among the groups(Fig. 3B and 3D), MAP at 10min in the Control group tended to be lower than that in other groups (Fig. 3B and 3D).

\subsection{Artificial oxygen pneumoperitoneum combined with abdominal lifting and compression did not alleviate or aggravate the impact of hypoxia}

To investigate the effects of hypoxia on hepatic and renal function, we assessed enzymes related to hepatic and renal function and oxidative stress at baseline, as well as at $2 \mathrm{~h}$ and $24 \mathrm{~h}$ after each experiment (Fig. 4). There were no differences in changes in ALT, AST, Crea, BUN, LDH, CK-MB, SOD, or MAO levels among groups. BUN, LDH, and SOD exhibited differences over time (Fig. 4D, 4E, 4G). After the experiment, BUN and LDH increased, while SOD decreased; however, all were within normal ranges. This indicates that the dogs had experienced hypoxic injuries, all of which were reversible. Moreover, the method did not appear to alleviate or aggravate this effect of hypoxia.

\section{Discussion}

In this study, we investigated a method of extra-pulmonary oxygenation via peritoneum: artificial oxygen pneumoperitoneum combined with abdominal lifting and compression. Overall, artificial 
oxygen pneumoperitoneum could prolong the hypoxia tolerance time of asphyxiated beagle dogs(Fig. 1B and 1C); its combination with abdominal lifting and compression could further increase $\mathrm{PaO}_{2}$ (Fig. 2A-C). Thus, this method may safely improve whole-body oxygenation status(Fig. 3 and 4).

Emergency airway and unpredictable difficult airway are not uncommon during anesthesia induction; these situations are extremely critical and difficult to manage appropriately. In addition, several case studies have reported the occurrence of complete airway obstruction during awake intubation ${ }^{[25]}$. Therefore, we aimed to identify other methods to supply oxygen promptly, particularly when encountered with above situations, in order to facilitate resuscitation, thereby providing additional time for tracheotomy. Previous studies have demonstrated that oxygen supply via peritoneum is feasible by direct abdominal ventilation or intraperitoneal infusion with oxygen carriers $\left.{ }^{[5,} 8,12,13,15-19\right]$.However, these methods are complex and cannot be performed immediately; therefore, they are not suitable for emergency resuscitation in the operating room.

Our method has both advantages and disadvantages compared with other peritoneal oxygenation methods. First, artificial pneumoperitoneum is a common technique that can be established rapidly in most hospitals, and abdominal lifting and compression is an easy-to-learn resuscitation procedure; this combination could be rapidly implemented to contribute to the establishment of invasive airway. Second, our experimental animals were beagle dogs, whose abdominal physiology is similar to that of humans; thus, the study is clinically applicable. Third, we adopted the Latin Square design, which enabled observation of the effects of different experimental times and individual dogs on the results. However, a disadvantage is that this method provided limited oxygen delivery, such that it increased $\mathrm{PaO}_{2}$ by $13 \mathrm{mmHg}$ at the 10 th min of asphyxia (Fig. 2C). However, a 13-mmHg increase in $\mathrm{PaO}_{2}$ in critically hypoxic patients may be critical for resuscitation.

In pre-experiments, we explored appropriate pressures for the artificial pneumoperitoneum, and found that excessive or minimal pressures could not improve the dogs' hypoxia tolerance. Hence, we chose a moderate pressure of $12 \mathrm{cmH}_{2} \mathrm{O}$ in this study. This pressure was appropriate 
for improving oxygenation status(Fig. 1C);possibly due to improved systemic oxygenation, our method may delay the onset of severe cardiac complications(Fig. 3A and 3B).

In this study, we used several criteria to determine when to terminate asphyxia and restore mechanical ventilation, which included $\mathrm{SpO}_{2}<50 \%$, MAP twice that of the baseline, or the occurrence of malignant arrhythmia. These were used to ensure thorough investigation and determine accurate pathological reversible time. To evaluate the suitability of these criteria, the recovery time and $\mathrm{PaO}_{2}$ at the time of asphyxia termination were analyzed; the results showed no differences among the four treatments(Fig. 1A and 1D). Furthermore, we assessed several biochemical parameters, including enzymes related to hepatic and renal function. We found that LDH, SOD, and BUN levels changed after anoxia; notably, these indexes were restored to normal within 24 hours(Fig. 4). Therefore, all dogs might have experienced a similar level of hypoxia, and the different asphyxia times could reflect variations in hypoxic tolerance.

There were some limitations in our study. To ensure animal safety, we only compared $\mathrm{PaO}_{2}$ at 9 and 10 min after asphyxia; we did not observe peritoneal pathological changes. Additionally, we did not explore methods to reducePaCO${ }_{2}$, and did not compare the occurrence of hypercapnia. Future studies should include investigation of the specific mechanism by which $\mathrm{PaO}_{2}$ was increased, methods to further improve $\mathrm{PaO}_{2}$, and the feasibility of this method in clinical practice.

\section{Conclusions}

This study found that artificial oxygen pneumoperitoneum, combined with abdominal lifting and compression, could safely improve systemic oxygenation; moreover, this method could prolong the hypoxia tolerance time of asphyxiated dogs. The potential clinical implication of this method is that, when accidental airway obstruction occurs, oxygen could be supplied in this manner, thereby facilitating resuscitation and increasing patient survival.

\section{Abbreviations}


$\mathrm{HR}$, heart rate;

MAP, mean artery pressure;

CO, cardio-output;

ALT, alanine aminotransferase;

AST, aspartate aminotransferase;

Crea, serum creatinine;

BUN, blood urea nitrogen;

Glu, blood glucose;

LDH, lactate dehydrogenase;

GGT, $\gamma$-glutamyl transpeptidase;

CK-MB, creatine kinase isoenzymes-B;

SOD, superoxide dismutase;

MAO, monoamine oxidase;

Hct, red blood cell specific volume;

Lac, lactate;

$B E$, buffer exces

\section{Declarations}

\section{Ethics approval}

The protocol of this study was approved by the Institutional Animal Care and Use Committee of the PLA General Hospital, Beijing, China(Chairperson Prof REN Guoquan) (Approval number: 
2015-D11-07). The necessity of this experiment and the rationality of animal species and quantity(eight female beagle dogs) were discussed and approved by the committee. The animals' welfare were concerned and adequate measures to minimize animals' pain and discomfort were taken by the authors. All procedures were performed in accordance with ethical guidelines published by International Council for Laboratory Animal Science (ICLAS).

\section{Consent for publication}

Not applicable.

\section{Availability of data and materials}

All data generated or analyzed during this study are presented within the additional supporting files.

\section{Competing interests}

The authors declare that they have no competing interests.

\section{Funding}

This study was conducted with the support of the National Natural Science Foundation of China (No. 81541114)(Funding body: CJB, who designed the study, collected and analyze the data) and the surface project of the Chinese PLA during the Twelfth Five-year Plan Period (CWS14J068)(Funding body: MWD, who designed the study, interpreted the data and took responsibility of the manuscript).

\section{Authors' Contributions}

CJB conceived the study, designed and carried out the experiment, and drafted the manuscript. ZYY participated in formulating the design of the studies, helped to implement the experiment, conducted the blood gas and biochemical analysis and drafted the manuscript. WW contributed to establish the animal model, monitor the vital signs and record the experimental data. LH contribute to perform the statistical analysis. MWD conceived of the study, participated in its design and coordination, secured funding for the project, and took overall responsibility for the work. All authors read and approved the final manuscript. 


\section{Acknowledgements}

Assistance with the study: We would like to thank the laboratory staff of the Experimental Animal Unit of PLA General Hospital for their unyielding support for the whole study, and we would like to thank Peng Bo for his tireless care for the beagles.

\section{Authors' Information}

$\mathrm{CJB}, \mathrm{PhD}$, chief physician(Anaesthesiology), deputy chief of Anaesthesia and Operation Centre of the Chinese PLA General Hospital, whose specialty are clinical anaesthesia, airway management and emergency resuscitation.

ZYY, MD, attending physician, anaesthetist of NO. 211 Hospital of Chinese PLA ; whose specialty are airway management, post-operative cognitive dysfunction prophylaxis, and postoperative analgesia.

WW, PhD, associated chief physician, anaesthetist of The General Hospital of the PLA Rocket Force.

$\mathrm{LH}, \mathrm{PhD}$, associated chief physician, anaesthetist of Anaesthesia and Operation Centre of the Chinese PLA General Hospital.

MWD, PhD, chief physician(Anaesthesiology), chief of Anaesthesia and Operation Centre of the Chinese PLA General Hospital, whose specialty are clinical anaesthesia, organ function protection during perioperative period, advanced life support and resuscitation to critically ill patients.

\section{References}

1. Soar J, Nolan JP: Airway management in cardiopulmonary resuscitation. Curr Opin Crit Care 2013, 19(3):181-187. 
2. Piepho T, Cavus E, Noppens R, Byhahn C, Dorges V, Zwissler B, Timmermann A: S1 guidelines on airway management : Guideline of the German Society of Anesthesiology and Intensive Care Medicine. Anaesthesist 2015, 64 Suppl 1:27-40.

3. Frerk C, Mitchell VS, McNarry AF, Mendonca C, Bhagrath R, Patel A, O'Sullivan EP, Woodall NM, Ahmad I: Difficult Airway Society $\mathbf{2 0 1 5}$ guidelines for management of unanticipated difficult intubation in adults. British journal of anaesthesia 2015, 115(6):827-848.

4. Neyrinck A: Management of the anticipated and unanticipated difficult airway in anesthesia outside the operating room. Current opinion in anaesthesiology 2013, 26(4):481-488.

5. Matsutani N, Takase B, Nogami Y, Ozeki Y, Ishihara M, Maehara T: The peritoneum as a novel oxygenation organ: revitalization of intraperitoneal oxygenation. Shock 2008, 30(3):250-253.

6. Gattinoni L, Carlesso E, Langer T: Clinical review: Extracorporeal membrane oxygenation. Crit Care 2011, 15(6):243.

7. Gentilello LM, Jurkovich GJ, Gubler KD, Anardi DM, Heiskell R: The intravascular oxygenator (IVOX): preliminary results of a new means of performing extrapulmonary gas exchange. J Trauma 1993, 35(3):399-404.

8. Zhang JY, Wang XH, Wang LJ, Xu B, Zheng M: Effect of oxygenation of transperitoneal ventilation on the death time after asphyxiation in rabbits. Minerva Anestesiolica 2010, 76(11):913-917.

9. Barrett CS, Bratton SL, Salvin JW, Laussen PC, Rycus PT, Thiagarajan RR: Neurological injury after extracorporeal membrane oxygenation use to aid pediatric cardiopulmonary resuscitation. Pediatr Crit Care Med 2009, 10(4):445-451.

10. Takagi S, Miyazaki S, Fujii T, Daikoku S, Sutani Y, Morii I, Yasuda S, Goto Y, Nonogi H: Dexamethasoneinduced cardiogenic shock rescued by percutaneous cardiopulmonary support (PCPS) in a patient with pheochromocytoma. Jpn Circ J 2000, 64(10):785-788.

11. Siriwardhana SA, Newfield AM, Lipton JM, Giesecke AH: Oxygen delivery by the peritoneal route. Canadian journal of anaesthesia 1990, 37(4 Pt 2):S159.

12. Barr J, Lushkov G, Strauss S, Gurevitch S, Lahat E, Bistritzer T, Klin B, Eshel G: Peritoneal ventilation in rabbits: augmentation of gas exchange with cisapride. Thorax 1996, 51(1):82-86.

13. Feshitan JA, Legband ND, Borden MA, Terry BS: Systemic oxygen delivery by peritoneal perfusion of oxygen microbubbles. Biomaterials 2014, 35(9):2600-2606.

14. Giffin DM, Gow KW, Warriner CB, Walley KR, Phang PT: Oxygen uptake during peritoneal ventilation in a porcine model of hypoxemia. Crit Care Med 1998, 26(9):1564-1568.

15. Kim WO, Cho CH: Transperitoneal oxygenation with lactated Ringer's solution. Yonsei Med J 1987, 28(1):34-37.

16. Matsutani N, Takase B, Nogami Y, Ozeki Y, Kaneda S, Maehara T, Kikuchi M, Ishihara M: Efficacy of peritoneal oxygenation using a novel artificial oxygen carrier (TRM-645) in a rat respiratory insufficiency model. Surg Today 2010, 40(5):451-455.

17. Marin T, Josephson CD, Kosmetatos N, Higgins M, Moore JE: Feeding preterm infants during red blood cell transfusion is associated with a decline in postprandial mesenteric oxygenation. J Pediatr 2014, 165(3):464-471.e461. 
18. Legband ND, Feshitan JA, Borden MA, Terry BS: Evaluation of peritoneal microbubble oxygenation therapy in a rabbit model of hypoxemia. IEEE Trans Biomed Eng 2015, 62(5):1376-1382.

19. Faithfull NS, Klein J, van der Zee HT, Salt PJ: Whole body oxygenation using intraperitoneal perfusion of fluorocarbons. British journal of anaesthesia 1984, 56(8):867-872.

20. McClung CD, Anshus AJ: Interposed Abdominal Compression CPR for an Out-of-Hospital Cardiac Arrest Victim Failing Traditional CPR. West J Emerg Med 2015, 16(5):690-692.

21. Li M, Song W, Ouyang YH, Wu DH, Zhang J, Wang LX, Li J: Clinical evaluation of active abdominal lifting and compression CPR in patients with cardiac arrest. Am J Emerg Med 2017, 35(12):1892-1894.

22. Agresta F, Campanile FC, Vettoretto N, Silecchia G, Bergamini C, Maida P, Lombari P, Narilli P, Marchi D, Carrara A et al: Laparoscopic cholecystectomy: consensus conference-based guidelines. Langenbecks Arch Surg 2015, 400(4):429-453.

23. Zhang S, Liu Q, Han S, Zhang Z, Zhang Y, Liu Y, Li J, Wang L: Standard versus Abdominal Lifting and Compression CPR. Evid Based Complement Alternat Med 2016, 2016:9416908.

24. Nolan JP, Soar J, Perkins GD: Cardiopulmonary resuscitation. BMJ 2012, 345:e6122.

25. Mushambi MC, Kinsella SM, Popat M, Swales H, Ramaswamy KK, Winton AL, Quinn AC: Obstetric Anaesthetists' Association and Difficult Airway Society guidelines for the management of difficult and failed tracheal intubation in obstetrics. Anaesthesia 2015, 70(11):1286-1306.

\section{Figures}



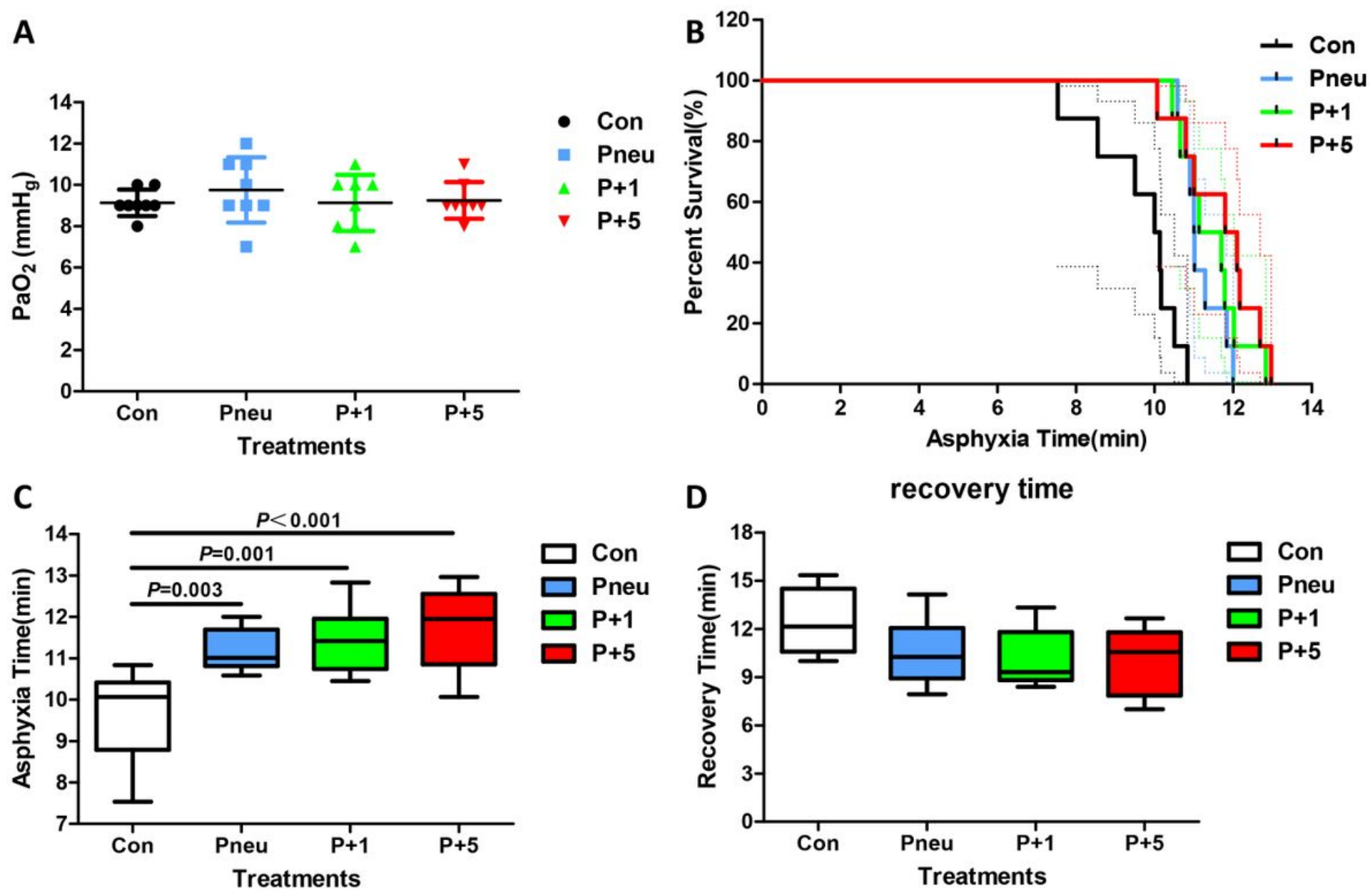

Figure 1

Asphyxia time, but not recovery time, of the Beagle dogs was lengthened by artificial oxygen pneumoperitoneum combined with abdominal lifting and compression. A)PaO2 values of each group were similar at the time of asphyxia termination. B) Asphyxia times of all four groups are illustrated by survival curves. C, D)Comparison of asphyxia(C) and recovery times (D) of the groups. Data are expressed as the mean \pm standard deviation in $A$, and mean $\pm 95 \%$ confidence interval in $C$ and $D$. $P=0.003$, Pneu group vs Control group, $\mathrm{P}=0.001, \mathrm{P}+1$ group vs Control group, $\mathrm{P} \otimes 0.001, \mathrm{P}+5$ group vs Control group. 


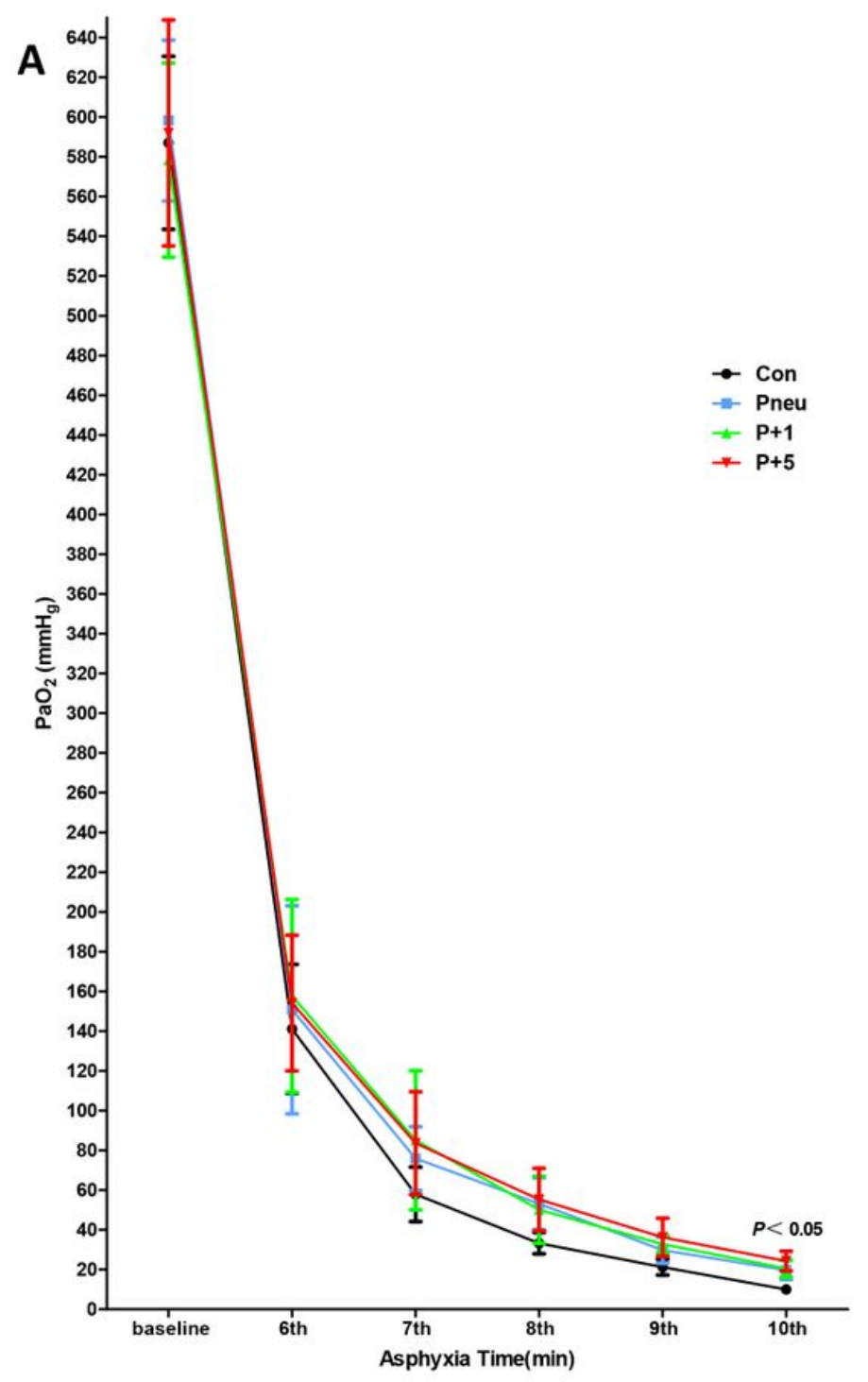

B

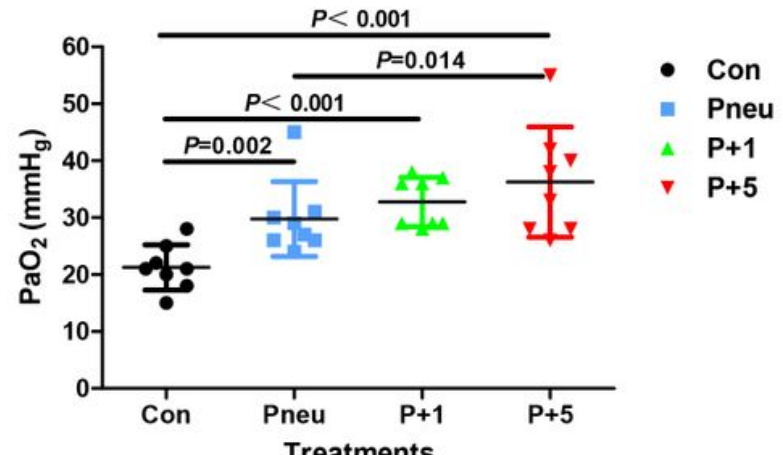

C
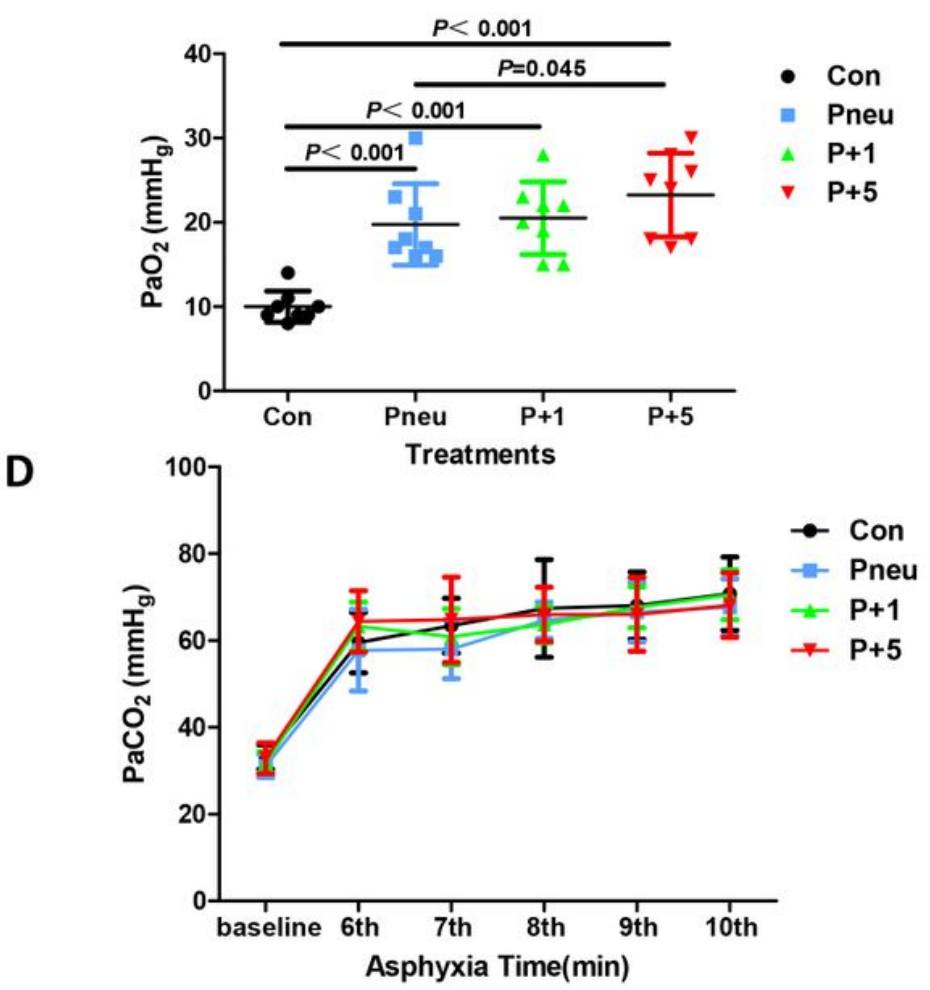

Figure 2

Artificial oxygen pneumoperitoneum combined with abdominal lifting and compression increased $\mathrm{PaO} 2$ of the Beagle dogs, but did not decrease PaCO2. A)PaO2 levels at baseline and at the 6th, 7th, 8th, 9th, and10th min of asphyxia of the four groups, showing a significant difference among groups $(P<0.05)$. $B, C)$ Comparison of $\mathrm{PaO} 2$ values at the 9th(B) and 10th(C) minutes within each group. D) Changes of PaCO2 levels from the baseline to the 10th min showed no significant differences among groups. Data are expressed as the mean \pm standard deviation. In $B) P=0.002$, Pneu group vs Control group $(P<0.001), P+1$ and $P+5$ group vs Control group, respectively, $(P=0.014), P+5$ group vs $P$ neu group; $I n C)(P<0.001) P n e u, P+1$ and $P+5$ group vs Control group, respectively $(P=0.045), P+5$ group vs Pneu group; 

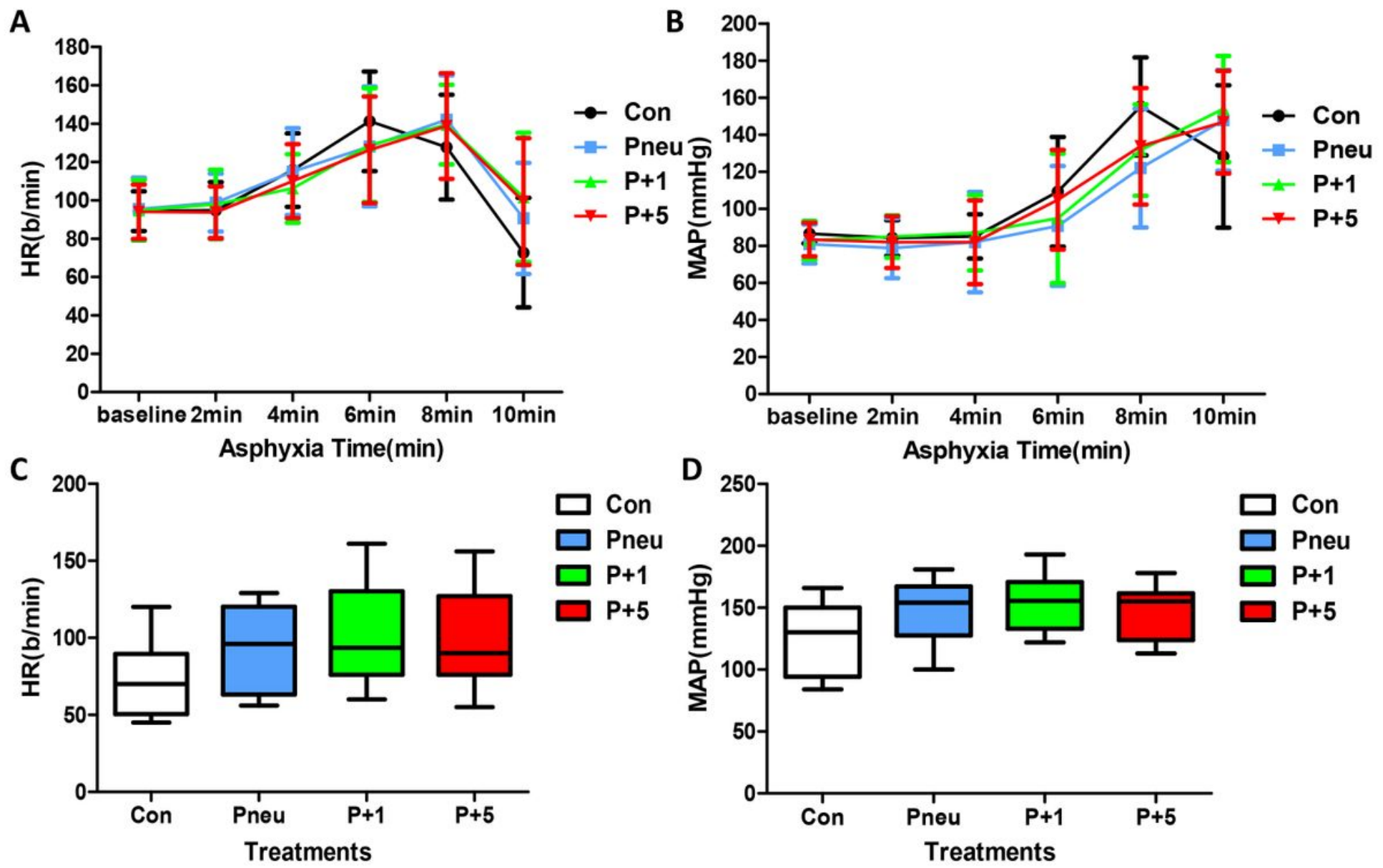

Figure 3

Changes in HR and MAP after the endotracheal tubes were clamped. $A$ and $B)$ Changes inHR(A) and MAP(B) of the four groups (shown every $2 \mathrm{~min}$ after asphyxia until $10 \mathrm{~min}$ ); $C$ and D) HR and MAP of the four groups at 10 min after asphyxia. Data are expressed as the mean \pm standard deviation in $A$ and $B$, and mean $\pm 95 \%$ confidence interval in $\mathrm{C}$ and $\mathrm{D}$. HR, heart rate; MAP, mean arterial pressure. 

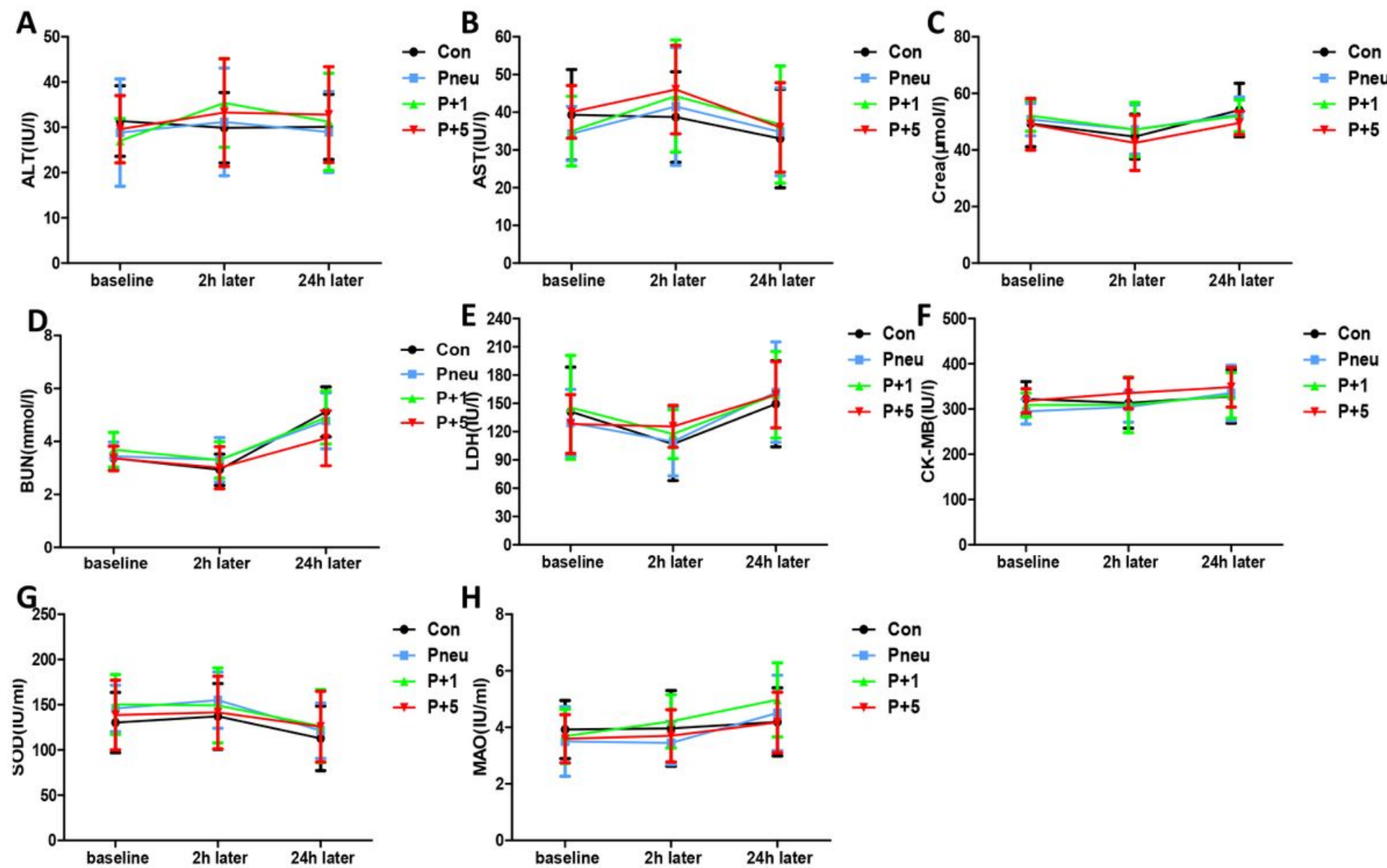

Figure 4

Artificial oxygen pneumoperitoneum combined with abdominal lifting and compression did not alleviate or aggravate the effects of hypoxia. A-H)Changes in ALT, AST, Crea, BUN, LDH, CK-MB, SOD, and MAO levels, respectively, in four groups at baseline, as well as at $2 \mathrm{~h}$ and $24 \mathrm{~h}$ following each experiment. All indexes showed no differences among groups(A-H), while BUN, $L D H$, and SOD showed differences over time (D,E,G). BUN and LDH increased24h after the experiment, while SOD decreased; however, all were within normal ranges. Data are expressed as the mean \pm standard deviation. ALT, alanine aminotransferase; AST, aspartate aminotransferase; Crea, serum creatinine; BUN, blood urea nitrogen; LDH, lactate dehydrogenase; CK-MB, creatine kinase isoenzymes-B; SOD, superoxide dismutase; MAO, monoamine oxidase

\section{Supplementary Files}

This is a list of supplementary files associated with this preprint. Click to download.

- ARRIVE.pdf

- Datasets.rar 